\section{AI-16 INTEGRATION OF BCR, TLR, BAFF RECEPTOR AND TACI SIGNALS PROMOTES AUTOANTIBODY PRODUCTION BY TRANSITIONAL B CELLS IN BAFF TRANSGENIC MICE}

${ }^{1}$ Du Samuel, 'Jacobs Holly M, 'Arkatkar Tanvi, 'Scharping Nicole, 1,2,3 Rawlings David J,

${ }^{1,2}$ Jackson Shaun $W^{*}$. 'Seattle Children's Research Institute, University of Washington School of Medicine, Seattle, WA, USA; ${ }^{2}$ Departments of Pediatrics, University of Washington School of Medicine, Seattle, WA, USA; ${ }^{3}$ Immunology, University of Washington School of Medicine, Seattle, WA, USA

\subsection{6/lupus-2018-Ism. 16}

Background The B cell survival cytokine BAFF has been linked with the pathogenesis of SLE. BAFF binds distinct B cell surface receptors, including the BAFF receptor (BAFF-R) and Transmembrane Activator and CAML Interactor (TACI). Although originally characterized as a negative regulator of $\mathrm{B}$ cell activation, TACI signals are critical for class-switched autoantibody production in BAFF transgenic (Tg) mice. Notably, while surface TACI expression is usually limited to mature B cells, we showed that excess BAFF promotes the expansion of TACI-expressing transitional B cells, with these cells representing an important source for class-switched autoantibodies in BAFF-Tg mice (Jacobs, et al. I Immunol, 2016). In the current study, we interrogate the signals required for transitional $B$ cell TACI expression and BAFF-driven autoantibody production.

Methods We first used a new 'fate-mapping' strategy to confirm that activated $\mathrm{TACI}^{+} \mathrm{B}$ cells falling within $\mathrm{CD} 21^{\text {lo }}$ transitional flow cytometry gates are bone fide transitional B cells. Subsequently, we interrogated the B cell signals required for transitional B cell TACI expression and antibody production using relevant murine genetic models crossed on the BAFF-Tg background.

Results To confirm that immature, transitional B cells are a prominant source for class-switched autoantibodies in BAFF-Tg mice, we developed a CD $21^{\text {Cre }}$.ROSA-YFP ${ }^{\text {fl/fl }}$ reporter strategy. By irreversibly labeling B cells that have expressed CD21, we confirmed that transitional B cells that had not yet differentiated beyond the T2 stage spontaneously produce class-switched autoantibodies in BAFF-Tg animals. We next determined the signals required for TACI upregulation on T1 transitional B cells. Surprisingly, signals downstream of B cell (BCR) and Toll-like (TLR) receptors exerted distinct impacts on transitional B cells. Whereas loss of BCR signals in Btk $k^{--}$.BAFF-Tg mice prevented transitional B cell TACI expression and resulted in loss of serum autoantibodies across immunoglobulin isotypes, lack of Myd88/TLR7 signals exerted a limited impact on autoantibody class switch recombination without impacting transitional B cell TACI expression. Moreover, in parallel with the protective effect of TACI deletion, loss of BAFF$\mathrm{R}$ activation signals protected against BAFF-driven autoimmunity. Notably, integration of all these signalling cascades (BCR, TACI, TLR7/MyD88, and BAFF-R) is required for production of pathogenic class-switched autoantibodies in BAFF-Tg mice, and for development of BAFF-driven lupus nephritis.

Conclusions In summary, we highlight how distinct signaling pathways integrate to promote class-switched autoantibody production by transitional B cells, findings with implications to the understanding of SLE pathogenesis and other humoral autoimmune diseases characterized by elevated serum BAFF.

\section{Al-17 RESPONSE GENE TO COMPLEMENT-32 EXPRESSION IS UPREGULATED IN LUPUS T CELLS AND PROMOTES IL- 17A EXPRESSION}

\footnotetext{
1,2Anamaria Talpos-Caia, ${ }^{2,3}$ Alexandru Tatomir, ${ }^{2,3}$ Vinh Nguyen, ${ }^{4}$ Cornelia D Cudrici, ${ }^{1}$ Simona Rednic, ${ }^{2,3}$ Horea G Rus, ${ }^{2,3}$ Violeta Rus*. 'University of Medicine and Pharmacy 'Iuliu Hatieganu', Cluj-Napoca, Romania; 'University of Maryland School of Medicine, Baltimore, USA; ${ }^{3}$ Veterans Administration Maryland Health Care System, Baltimore, USA; ${ }^{4}$ NIAMS, National Institutes of Health, Bethesda, USA
}

\subsection{6/lupus-2018-Ism.17}

Background RGC (Response Gene to Complement)-32 is a cell cycle regulator widely expressed in normal tissues including brain, kidney, spleen, thymus, multiple tumors and in a variety of cell lines. RGC-32 is localized in the cytoplasm and translocates to the nucleus upon upregulation by complement activation, growth factors and cytokines. RGC-32 is induced by TGF $\beta$ in fibroblasts, astrocytes and human renal proximal tubular cells and mediates TGF $\beta$ dependent profibrotic pathways. RGC-32 is preferentially upregulated in murine Th17 cells and promotes their differentiation. Patients with Systemic Lupus Erythematosus (SLE) display increased serum levels, expanded frequency of IL-17 producing cells. Whether RGC32 plays a role in human Th17 differentiation pathway and in Th17 abnormalities in lupus patients has not yet been investigated.

Methods RGC-32 expression in naïve $\mathrm{CD}^{+}{ }^{+} \mathrm{T}$ cells from normal controls stimulated with cytokines alone or under Th0, Th1, Th2, Th17 and Treg conditions was determined by flow cytometry and RT-PCR. RGC-32 mRNA expression in PBMCs of lupus patients was assessed with the Autoimmune Disease Profiling cDNA Array spotted with cDNA from $\mathrm{CD}^{+}$, $\mathrm{CD}_{1}{ }^{+}$and $\mathrm{CD} 14^{+}$cells and by RT-PCR and flow cytometry. RGC-32 nuclear translocation after stimulation under Th17 conditions was assessed by Western blotting. RGC-32 overexpression and silencing was performed by nucleofection and the effect on IL-17A mRNA levels in $\mathrm{CD}^{+} \mathrm{T}$ cells under Th17 conditions was determined by RT-PCR.

Results RGC-32 mRNA expression was upregulated by TCR stimulation and TGF $\beta$ and was more robust under Th17 (3.2 \pm fold) and Treg $(2.6 \pm 0.8$ fold $)$ vs Th1 (1.3 \pm 0.4 fold) and Th2 (1.8 \pm 0.1 fold) conditions. Moreover, upon stimulation with anti-CD3/CD28 and TGF $\beta$, RGC-32 was translocated into the nucleus. Other cytokines such as IFN $\alpha$, IL- $1 \beta$, TNF $\alpha$ did not upregulate RGC-32 mRNA either alone or in combination with TCR stimulation. Overexpression or silencing of RGC-32 in $\mathrm{CD}^{+}{ }^{+} \mathrm{T}$ cells upregulated, respectively downregulated IL-17A transcript levels and protein secretion. RGC-32 mRNA and protein level were significantly increased in $\mathrm{CD}_{19}{ }^{+} \mathrm{B}$ cells and $\mathrm{CD} 3^{+} \mathrm{T}$ from lupus patients compared to controls.

Conclusions These results suggest that RGC-32 promotes the differentiation of human Th17 cells. Furthermore, T cells from patients with SLE exhibit increased expression of RGC32 compared to controls. These data support the idea that RGC-32 signaling may enhance disease expression in SLE by promoting abnormalities in the Th17 pathway and provide a compelling rationale for further investigating the therapeutic potential of blocking RGC-32 in SLE. 